\title{
Integración de Un Sistema Robótico de Terapia Ocupacional para Extremidades Superiores con Estimulación Visual/Táctil de Los Pacientes
}

\author{
Integration of an Occupational-Therapy Robotic System for Upper Limbs with \\ Visual/tactile Stimulation of Patients
}

\author{
C. A. Vidrios-Serrano, B. R. Maldonado-Fregoso, I. Bonilla-Gutiérrez, M. O. Mendoza-Gutiérrez, E. J. González-Galván \\ Universidad Autónoma de San Luis Potosí
}

\section{RESUMEN}

Los ejercicios tradicionales de rehabilitación de extremidades superiores tienen como objetivo principal recuperar la fuerza o rango de movimiento del área lesionada de los pacientes. Una opción alternativa que se ha presentado en los últimos años es el uso de interfaces hápticas, las cuales han mostrado ser herramientas potenciales en el apoyo de las terapias de rehabilitación. En este artículo se presenta un sistema de rehabilitación háptico de movimientos finos en extremidades superiores, cuya característica principal es que los usuarios del sistema pueden interactuar de forma visual y táctil con objetos virtuales mezclados con escenarios reales logrando con ello un ambiente de realidad aumentada. El sistema fue probado en dos etapas, ambas con sujetos que presentaban un grado de discapacidad en extremidades superiores. Los datos recopilados fueron trayectorias seguidas, errores de seguimiento y la actividad muscular obtenida por medio de electromiografía; esta información recolectada permitió analizar de forma cuantitativa el grado de avance de los pacientes. Además, se consideraron las valoraciones hechas por fisioterapeutas, concluyendo que el sistema propuesto puede ser utilizado como una herramienta viable que complementa a las terapias de rehabilitación convencionales.

PALABRAS CLAvE: dispositivo háptico, realidad aumentada, terapias de rehabilitación 


\section{ABSTRACT}

Traditional upper limb rehabilitation exercises are primarily aimed at regaining the strength or range of motion of the patients' injured area. An alternative option that has been presented in the last years is the use of haptic interfaces, which have shown their potential as tools that support rehabilitation therapies. This article presents a haptic system of rehabilitation for fine upper limb movements, whose main characteristic is that users of the system can interact in a visual and tactile fashion with virtual objects mixed with real scenarios, thereby achieving an augmented reality environment. The system was tested in two stages, both with subjects who had a degree of disability in upper limbs. The data collected were followed trajectories, follow-up errors and the muscular activity obtained by means of electromyography; the collected information enabled the analysis, in a quantitative way, of the degree of progress of the patients. In addition, the assessments made by physiotherapists were considered, concluding that the proposed system can be used as a viable complementary tool for conventional rehabilitation therapies.

KEYWORDS: haptic device, augmented reality, rehabilitation therapies

\section{Correspondencia}

DESTINATARIO: Isela Bonilla Gutiérrez

INSTITUCIÓN: Universidad Autónoma de San Luis Potosí DIRECCIÓN: Av. Salvador Nava S/N, Zona Universitaria,

C.P. 78290, San Luis Potosí, S.L.P., México

CORREO ELECTRÓNICO: isela.bonilla@uaslp.mx

\section{Fecha de recepción:}

29 de septiembre de 2017

Fecha de aceptación:

06 de marzo de 2018 


\section{INTRODUCCIÓN}

Según la Organización Mundial de la Salud (OMS), en la actualidad hay aproximadamente mil millones de personas en el mundo con alguna discapacidad, la mayoría de ellas sin acceso a algún tipo de rehabilitación ${ }^{[1]}$. En México, el Instituto Nacional de Estadística y Geografía (INEGI) reportó que la prevalencia de personas con discapacidad es de aproximadamente $6 \%$ de la población, esto es, unos 7.5 millones de personas, de los cuales al menos una tercera parte tiene alguna discapacidad en sus extremidades superiores ${ }^{[2]}$.

Las causas principales por las cuales las personas no cuentan con acceso a servicios de rehabilitación son la escasez de personal capacitado para brindar terapias de rehabilitación y el elevado costo de las mismas, así como el creciente número de personas que requieren de dichas terapias. Por mencionar algunos datos, el organismo reportó en 2006 que en los países en vías de desarrollo, como México, había solamente 1,500 terapeutas para atender a una población de 125,000,000 habitantes ${ }^{[3]}$.

Con relación a los costos económicos, la OMS afirmó que es difícil cuantificarlos, puesto que el impacto que manifiesta la discapacidad en la economía de los países es de origen multifactorial, y los datos con los que se cuenta son aquellas cifras reportadas por los países pertenecientes a la Organización para la Cooperación y el Desarrollo Económicos (OCDE), los cuales destinan, en promedio, el $1.2 \%$ del producto interno bruto (PIB) a programas de salud que están dirigidos a personas con discapacidad.

A pesar de los altos montos destinados a programas de apoyo a personas con discapacidad, la OMS reportó en 2011 que en países considerados con ingresos altos, como Canadá o Nueva Zelanda, entre el 20\% y el $40 \%$ de este grupo de la población no tienen cubiertas sus necesidades de asistencia, situación que se agrava considerablemente en países en vías de desarrollo ${ }^{[1]}$.
En años recientes, los sistemas de rehabilitación robótica han surgido como un complemento a las terapias de rehabilitación tradicional, los cuales se plantean como mecanismos de ayuda para aquellos países en donde el personal para proveer terapias de rehabilitación convencionales es insuficiente e incluso se piensa que a largo plazo esta tecnología pueda reducir los costos de las terapias de rehabilitación.

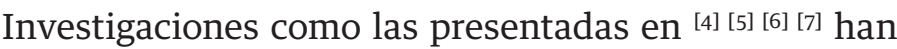
demostrado que las terapias de rehabilitación robótica son efectivas como método de rehabilitación o como un complemento a las terapias de rehabilitación convencionales.

Una de las principales cualidades de los sistemas de rehabilitación robótica es que éstos pueden proveer la cantidad de repeticiones que se desee de una terapia sin disminuir la calidad de la misma, siendo ésto de gran utilidad para los usuarios, ya que diversos estudios han demostrado que la recuperación de pacientes con problemas de motricidad causados por un Evento Cerebro-Vascular (ECV) o algún otro tipo de daño cerebral, está directamente relacionada con el número de repeticiones que el paciente realice en su terapia ${ }^{[8] ~[9] . ~}$ Asimismo, se ha estudiado la correlación que existe entre la velocidad de recuperación de un paciente que ha sufrido un daño cerebral y los estímulos sensoriales que recibe el paciente combinados con su terapia física, por lo que el uso de estímulos táctiles, auditivos y/o visuales aceleran la recuperación del mismo ${ }^{[10]}$ [11] [12] $^{\text {. }}$

Diversos grupos de investigación han hecho uso de las características antes mencionadas para el diseño de sistemas de rehabilitación basados en realidad virtual, en los trabajos presentados en ${ }^{[13]}$ [14] [15] $^{\left[{ }^{10}\right.}$ demuestra la efectividad de dichos sistemas como complemento a las terapias de rehabilitación convencionales. En estas investigaciones se hace una comparativa entre grupos de pacientes que reciben terapias de rehabilitación convencionales y grupos de pacientes que complementan dichas terapias con actividades basadas en 
realidad virtual, en la mayoría de los casos el grado de rehabilitación resultó mayor para aquellos que utilizaron los sistemas de realidad virtual, lo que valida el potencial de estos sistemas.

Otra forma de utilizar los estímulos sensoriales como mecanismo de rehabilitación es por medio de los robots hápticos, los cuales han surgido como una opción útil para proveer terapias de rehabilitación. Gracias a la flexibilidad de éstos, es posible implementar en ellos plataformas de rehabilitación con características muy variadas, y dado que este tipo de robots son programados para que el usuario pueda tener la sensación de tocar objetos virtuales, los pacientes que reciben terapias con este tipo de sistemas, son estimulados de manera visual y táctil, lo que favorece su recuperación.

Los sistemas hápticos han sido utilizados en diversos trabajos de investigación, pudiendo destacarse el trabajo presentado por Chiang et. al. en ${ }^{[16]}$, en donde simulan en un ambiente de realidad virtual la realización de tareas cotidianas con retroalimentación de fuerza por medio de una interfaz háptica. El sistema fue probado por 20 sujetos con alguna discapacidad, mostrando mejoría en varios aspectos en los que fueron evaluados después de varias sesiones de rehabilitación. En el trabajo presentado por Turolla et. al. en ${ }^{[17]}$, se hace uso de los robots hápticos en un entorno de realidad virtual como medio para la rehabilitación de muñeca. En pruebas clínicas realizadas con 15 pacientes se observó que todos ellos mostraron algún nivel de mejoría después de varias sesiones de terapia con este sistema.

Por otra parte, algunos investigadores hacen uso de la realidad aumentada, la cual es definida como la visión de un entorno físico del mundo real mezclada con elementos virtuales, creando una realidad mixta en tiempo real ${ }^{[18]}$. Hossain et. al. proponen en ${ }^{[19]}$ un conjunto de juegos "serios" desarrollados en un entorno de realidad aumentada, diseñados y probados en pacientes con diferentes tipos de dificultades en las extremidades superiores, después de cierto número de sesiones de rehabilitación todos los pacientes mostraron mejoras en evaluaciones cuantitativas realizadas.

Por su parte Dvorkin et. al. en su trabajo ${ }^{[20]}$ utilizan un robot háptico para rehabilitación de extremidades superiores. Mediante el sistema háptico es posible sentir el contacto con objetos virtuales proyectados en un sistema de realidad aumentada llamado PARIS (Personal Augmented Reality Immersive System), el cual es una pantalla semitransparente donde se proyectan los objetos virtuales y gracias a la transparencia de la misma es posible observar las manos del usuario a través de ella.

Una de las características deseadas y de mayor importancia en sistemas de rehabilitación robótica, es poder analizar de forma cuantitativa la evolución o progreso de los pacientes durante el proceso de terapia. Además de los parámetros o índices que pueden ser obtenidos directamente de los componentes del sistema robótico, algunos grupos de investigación han hecho uso de la caracterización de señales de electromiografía (EMG) para determinar parámetros como: fortalecimiento muscular en el caso del trabajo de Phinyomark et. al. en ${ }^{[21]}$, fatiga muscular estudiada por Cifrek et. al. en ${ }^{[22]}$, identificación de movimientos de alguna extremidad en ${ }^{[23]} \mathrm{o}$ incluso como método de activación y control de prótesis robóticas ${ }^{[24]}$.

Desarrollar sistemas de rehabilitación que mezclen escenarios de realidad aumentada con sistemas hápticos, sin duda ayudará a mejorar los procesos de rehabilitación tradicionales. La flexibilidad que presentan estos sistemas permite cambiar y/o modificar el grado de dificultad del juego, así como, presentar al usuario su grado de avance mediante evaluaciones diarias, logrando de esta forma avances significativos en su recuperación motriz, y evitando que el usuario pierda la motivación e interés en su proceso de rehabilitación. 
En este sentido, se presenta un sistema para rehabilitación de movimientos finos de extremidades superiores, donde se estimula al usuario de forma visual y táctil. El entorno fue desarrollado utilizando realidad aumentada, el usuario utiliza un sistema de inmersión y de esta forma se logra que visualice un entorno real mezclado con objetos virtuales. Para que el usuario pueda interactuar con los objetos virtuales, el sistema incluye un robot háptico además de contar con distintos métodos de evaluación cuantitativa del progreso de los pacientes durante la terapia ${ }^{[25][26][27]}$.

\section{METODOLOGÍA}

La metodología por medio de la cual se desarrolló este proyecto se divide en dos secciones principales las cuales se describen a continuación.

\section{Sistema de aumento de la realidad}

El aumento de la realidad consiste en capturar imágenes de un entorno real para mezclarlas con elementos virtuales de una forma coherente, para dicha tarea en este proyecto se ha elegido la metodología de detección de marcadores. Un marcador es una figura de colores y formas muy distintivas, como la mostrada en la Figura 1, que pueda ser detectada fácilmente y en tiempo real por un sistema de visión por computadora; este proceso de detección se realiza para tomar estos marcadores como referencia de la posición y orientación (postura) que tomarán los objetos virtuales introducidos en la escena.

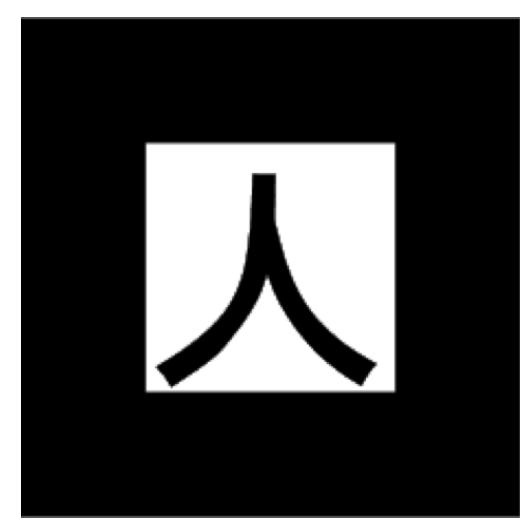

FIGURA 1. Ejemplo de marcador.
Para este proyecto se utiliza la librería de programación ARToolKit, la cual está diseñada para realizar el proceso de aumento de la realidad, este procedimiento consta de cuatro tareas fundamentales:

(i) captación de la escena, (ii) identificación de la escena, (iii) mezclado de la realidad y aumento, y (iv) visualización. A continuación se detallan cada una de estas tareas.

\section{Captación de la escena}

El proceso de captación de la escena es básicamente un cambio de coordenadas de la representación de un objeto en el mundo real (tridimensional/3D) y su proyección correspondiente en el plano de la imagen captada por una cámara (bidimensional/2D), esta transformación es conocida como proyección en perspectiva y es muy utilizada ya que el ojo humano funciona de forma similar, donde una de las principales características de este tipo de proyección es que los objetos lucen más pequeños entre más alejados estén del observador.

La posición en la cual se proyecta un objeto (3D) en una imagen (2D) está determinada principalmente por la posición de dicho objeto en el espacio, sin embargo, esta proyección también se ve afectada por las características de construcción de la cámara con la cual es captada la escena, conocidas como "parámetros intrínsecos de la cámara"' y que son representados de forma matricial como se muestra en la Ecuación (1).

$$
K=\left(\begin{array}{ccc}
\propto_{u} & S_{k} & u_{0} \\
0 & \propto_{v} & \mid v_{0} \\
0 & 0 & 1 / f
\end{array}\right)
$$

donde $\propto_{u} \mathrm{y} \propto_{v}$ son factores de escalamiento de la imagen en los ejes $x$ y $y$ respectivamente, $S_{k}$ es conocido como el sesgo o inclinación de los pixeles de la imagen, $u_{0} \mathrm{y} v_{0}$ representan la coordenada central de la imagen, también conocida como punto principal y $f$ representa la distancia focal de la cámara. Por lo tanto, el modelo 
de proyección en perspectiva puede ser escrito como se observa en la Ecuación (2).

$$
\left(\begin{array}{l}
u \\
v \\
1
\end{array}\right)=\left(\begin{array}{ccc}
\propto_{u} & S_{k} & u_{0} \\
0 & \propto_{v} & v_{0} \\
0 & 0 & 1 / f
\end{array}\right)\left(\begin{array}{l}
x \\
y \\
z
\end{array}\right)
$$

donde $x, y$ y $z$ representan las coordenadas de un objeto en el espacio, y las coordenadas de dicho objeto proyectado en la imagen son representadas por $u$ y $v^{[28]}$.

\section{Identificación de la escena}

Una vez que se ha capturado la imagen con una cámara, es necesario identificar el marcador dentro de la escena captada, haciendo uso de una serie de algoritmos computacionales. Este proceso puede resumirse en los siguientes pasos:

1. Cambiar el espacio de color de la imagen a escala de grises (ver Figura 2(a)), con la finalidad de mejorar el contraste de la imagen.

2. Binarización de la imagen: en una imagen en escala de grises, representada con 8 bits, cada pixel toma cualquier valor de intensidad entre $0 \mathrm{y}$ 255; el proceso de binarización consiste en cambiar estos valores de intensidad de cada pixel a 0 (negro) o 1 (blanco), por lo tanto, es necesario establecer un valor de umbral y compararlo con el valor de cada uno de los pixeles de la imagen en gris, si el valor está por encima del umbral se cambia su valor a 0 y si está por debajo cambia su valor a 1 , con esto se obtiene una imagen binarizada como la mostrada en la Figura 2(b).

3. Detección de bordes en la imagen: consiste en localizar las regiones donde se encuentre un pixel blanco junto a uno negro, el resultado se muestra en la Figura 2(c). Dado que los marcadores que se quieren detectar son cuadrados, se utiliza la transformada Hough ${ }^{[29]}$ para detectar líneas rectas en la imagen y tomar como candidatos a ser un marcador aquellas áreas delimitadas por cuatro líneas rectas de longitud similar. En la Figura 2(d) se indican en color rojo aquellas áreas que son candidatas a ser un marcador y en color verde las líneas rectas detectadas que no cumplen con las características necesarias para ser un marcador.

4. Normalización: este proceso consiste en obtener una representación de vista frontal (también conocida como posición fronto-paralela) del área de un candidato a ser marcador, la cual nos ayudará a compararla con una imagen patrón para determinar si la figura es identificada o no como el marcador que se está buscando ${ }^{[30]}$.

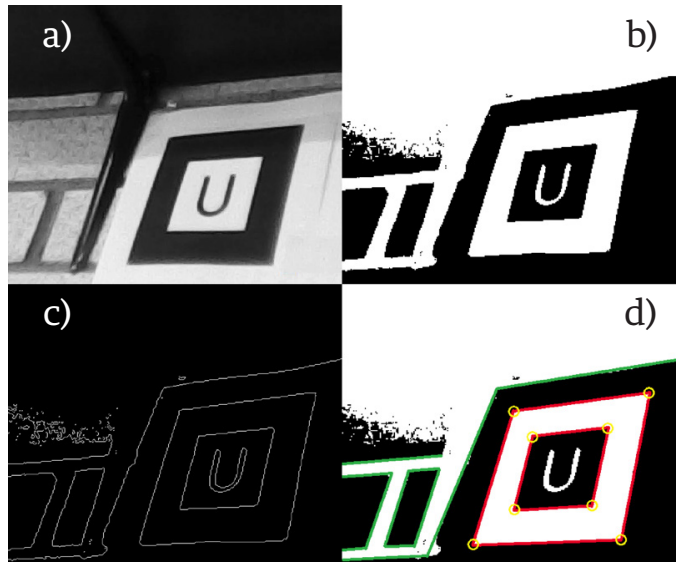

FIGURA 2. Proceso de detección de candidatos.

(a) Imagen en escala de gris, (b) Imagen binarizada,

(c) Imagen de bordes y (d) Imagen con candidatos.

Para llevar a cabo el proceso de normalización, supongamos que se conocen las coordenadas $X_{i}$ de las cuatro esquinas del marcador en el espacio 3D, y las coordenadas $x_{i}$ de las cuatro esquinas del marcador proyectadas en la imagen 2D. Por lo tanto, la posición en el espacio de la cámara es calculada mediante la matriz de proyección $P$, como se muestra en la Ecuación (3).

$$
P=K[R \mid t]
$$

donde $R$ es una matriz de $3 \times 3$ que representa la rotación de la cámara con respecto al marcador y $t$ es un 
vector de tres elementos que representa la traslación de la cámara con respecto al marcador. Por lo tanto cada uno de los puntos $x_{i}$ se relacionan con sus correspondientes $X_{i}$ por medio de la Ecuación (4).

$$
x_{i} \sim \lambda P X_{i}
$$

donde $\lambda$ es un factor de escala.

Si se sustituye la Ecuación (3) en (4) y se escribe la matriz de rotación en función de cada una de sus columnas $R=\left(r_{1} r_{2} r_{3}\right)$ se obtiene la ecuación (5)

$$
\left(\begin{array}{l}
u \\
v \\
1
\end{array}\right) \sim \lambda K\left(\begin{array}{llll}
r_{1} & r_{2} & r_{3} & t
\end{array}\right)\left(\begin{array}{l}
x \\
y \\
z \\
1
\end{array}\right)
$$

Ahora bien, como se está buscando obtener la posición de la cámara con respecto al marcador, se puede considerar que el centro del marcador tiene coordenadas $(0,0,0)$. Por lo tanto, ya que el marcador es plano la coordenada $z=0$ para todo el marcador, entonces se pueden re-escribir las coordenadas del marcador en el espacio para todos sus puntos como $X_{i}=(x, y, 0,1)$, por lo que la Ecuación (5) se puede reescribir como se muestra en la Ecuación (6), donde la matriz $H$ de $3 \times 3$, es conocida como matriz de homografía y relaciona los puntos $X_{i}$ del plano del marcador con los puntos del plano de la imagen $x_{i}$.

$$
\begin{aligned}
\left(\begin{array}{l}
u \\
v \\
1
\end{array}\right) & \sim \lambda K\left(\begin{array}{llll}
r_{1} & r_{2} & r_{3} & t
\end{array}\right)\left(\begin{array}{l}
x \\
y \\
0 \\
1
\end{array}\right) \\
& =\lambda K\left(\begin{array}{lll}
r_{1} & r_{2} & t
\end{array}\right)\left(\begin{array}{l}
x \\
y \\
1
\end{array}\right) \\
& =\lambda H\left(\begin{array}{l}
x \\
y \\
1
\end{array}\right)
\end{aligned}
$$

Con base en la consideración de que las coordenadas del centro del marcador son $(0,0,0)$ y el conocimiento de las dimensiones del marcador, es posible obtener las coordenadas $(x, y, z)^{T}$ de cada una de sus esquinas. De igual forma, es posible conocer las coordenadas de cada una de las esquinas del marcador proyectadas en la imagen 2D. Por lo tanto, si se desarrolla la Ecuación (6) para la primera esquina del marcador se obtienen las Ecuaciones (7) y (8)

$$
\begin{aligned}
& u\left(h_{31}+h_{32} y+h_{33}\right)=h_{11} x+h_{12} y+h_{13} \\
& v\left(h_{31}+h_{32} y+h_{33}\right)=h_{21} x+h_{22} y+h_{23}
\end{aligned}
$$

Si de igual forma se calculan las correspondencias para las otras tres esquinas del marcador, se obtiene un sistema de ecuaciones $A h=0$ donde $h$ son los componentes $h_{i j}$ acomodados en forma de vector de nueve elementos y $A$ está dada por la Ecuación (9)

$$
A=\left(\begin{array}{ccccccccc}
x_{1} & y_{1} & 1 & 0 & 0 & 0 & -u_{1} x_{1} & -u_{1} y_{1} & -u_{1} \\
0 & 0 & 0 & x_{1} & y_{1} & 1 & -v_{1} x_{1} & -v_{1} y_{1} & -v_{1} \\
x_{2} & y_{2} & 1 & 0 & 0 & 0 & -u_{2} x_{2} & -u_{2} y_{2} & -u_{2} \\
0 & 0 & 0 & x_{2} & y_{2} & 1 & -v_{2} x_{2} & -v_{2} y_{2} & -v_{2} \\
x_{3} & y_{3} & 1 & 0 & 0 & 0 & -u_{3} x_{3} & -u_{3} y_{3} & -u_{3} \\
0 & 0 & 0 & x_{3} & y_{3} & 1 & -v_{3} x_{3} & -v_{3} y_{3} & -v_{3} \\
x_{4} & y_{4} & 1 & 0 & 0 & 0 & -u_{4} x_{4} & -u_{4} y_{4} & -u_{4} \\
0 & 0 & 0 & x_{4} & y_{4} & 1 & -v_{4} x_{4} & -v_{4} y_{4} & -v_{4}
\end{array}\right)
$$

A partir de la expresión (9) se tiene un sistema homogéneo de ocho ecuaciones y nueve incógnitas, por lo que para resolverlo se puede utilizar factorización SVD (Singular Value Decomposition) ${ }^{\text {[31] }}$ o asignarle un valor a alguna de las incógnitas $h_{i j}$ y encontrar la solución del nuevo sistema de ecuaciones, con lo cual se habrá encontrado la matriz $H$.

Una vez calculada $H$ y asumiendo que $K$ es conocida, ya que estos parámetros pueden ser calculados fuera de línea o pueden ser provistos por el fabricante de la cámara, y recordando que $H=K\left(r_{1} r_{2} t\right)$ entonces se tiene que $\left(r_{1} r_{2} t\right)=K^{-1} H$, dado que la matriz de rotación $\mathrm{R}$ tiene columnas ortonormales entre sí, es posible calcular $r_{3}=r_{1} \times r_{2}$. Por lo tanto, la matriz de proyección puede ser calculada con la ecuación (10). 


$$
P=K\left[\begin{array}{llll}
r_{1} & r_{2} & \left(r_{1} \times r_{2}\right) & t
\end{array}\right]
$$

Como se mencionó previamente, la matriz de homografía $H$ sirve para relacionar las coordenadas del plano del marcador con las coordenadas del plano de la imagen, por lo que a partir de la imagen capturada con la cámara, sin importar la posición y orientación que ésta tenga, es posible hacer una reconstrucción de cómo se ve el marcador en posición fronto-paralela. Esto se logra con la Ecuación (11)

$$
X_{i j}=H^{-1} x_{i j}
$$

Donde $x_{i j}$ es el valor que tiene el pixel de la imagen 2D en la posición ij. Si se aplica la Ecuación (11) a cada uno de los pixeles de la imagen presentada en la Figura 2(d), se obtiene una imagen normalizada del marcador como se muestra en la Figura 3.

Por último se realiza el cálculo de la correlación normalizada entre la imagen normalizada y una imagen patrón del marcador a detectar, si el valor de correlación se encuentra por arriba de un valor de umbral previamente establecido se considera una detección positiva.

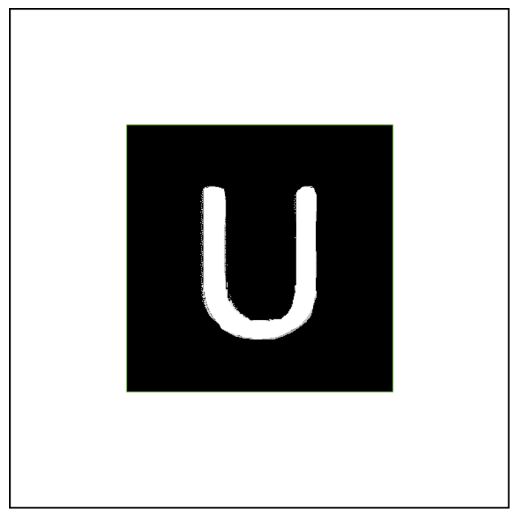

FIGURA 3. Imagen normalizada.

\section{Mezclado de la realidad y aumento}

Una vez que se ha detectado un marcador en la escena que servirá como referencia para la proyección de objetos virtuales, es necesario el uso de librerías de progra- mación gráfica para generar dichos objetos, en este caso se eligió la librería de programación OpenGL.

Una de las principales características de OpenGL, es que esta librería permite trabajar con objetos virtuales en un espacio $3 \mathrm{D}$, por lo que es posible crear objetos virtuales con coordenadas similares a las del mundo real y OpenGL se encarga de hacer la conversión a 2D para su posterior visualización.

De igual manera, en el sistema de referencia del espacio virtual se considera que el centro del marcador detectado tiene coordenadas $(0,0,0)$ y éste se toma como referencia para introducir los objetos virtuales. Además, previamente se calculó la matriz de proyección, la cual determina la posición de la cámara con la que fue capturada la imagen, por lo tanto haciendo uso de esta misma matriz de proyección se puede, en el espacio virtual, posicionar una cámara también virtual desde donde OpenGL visualizará los objetos virtuales agregados en la escena. En conclusión, la librería de programación nos ayuda a superponer un espacio virtual sobre el espacio real, ambos en 3D con las mismas coordenadas, logrando la fusión de ambos escenarios. Asimismo, se coloca una cámara virtual en la misma posición que la cámara real, con la finalidad de que ambos escenarios, real y virtual, sean observados desde el mismo punto, por lo tanto habrá una fusión completa y coherente del escenario real y virtual y se habrá conseguido el aumento de la realidad.

\section{Visualización}

Como medio de visualización se eligieron unos lentes de inmersión, que al igual que la cámara con la que se capturan las imágenes, son de alta definición. En la Figura 4 se muestra la manera en que se colocan la cámara y los lentes sobre la cabeza del usuario, mediante esta configuración el paciente visualiza el mundo a través de los lentes de la misma forma que lo haría si no los usara, con la ventaja de que con este sistema percibe la realidad aumentada. 


\section{Interacción háptica}

Una característica muy importante de este sistema de terapia es la posibilidad que tiene el usuario de interactuar de forma táctil con los objetos virtuales introducidos en el escenario real. Para este fin se hace uso de un robot háptico, el cual cuenta con las características necesarias para implementar el sistema táctil que simule los efectos físicos de la interacción entre el usuario y los objetos virtuales.

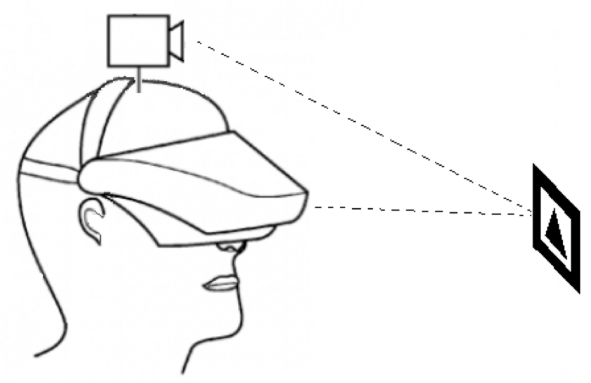

FIGURA 4. Posición de la cámara y los lentes.

Para conseguir la simulación de contacto por parte del robot háptico es necesario calcular la posición en el espacio del efector final del robot a partir del conocimiento de la posición de sus articulaciones, esto se logra gracias al modelado cinemático ${ }^{[32]}$ expresado en la Ecuación (12)

$$
\begin{gathered}
T=\operatorname{Tras}\left(y, d_{1}\right) \operatorname{Tras}\left(z, d_{2}\right) \operatorname{Rot}\left(x,-q_{0}\right) \operatorname{Rot}\left(x, q_{1}\right) \\
\operatorname{Tras}\left(z,-l_{0}\right) \operatorname{Rot}\left(x, q_{2}\right) \operatorname{Tras}\left(z,-l_{1}\right)
\end{gathered}
$$

donde $d_{1}$ y $d_{2}$ representan un offset en el origen del sistema coordenado del robot en los ejes $y$ y $z$ respectivamente, $q_{0}, q_{1}$ y $q_{2}$ representan los ángulos de giro de cada una de las primeras tres articulaciones y $l_{0}$ y $l_{1}$ son las longitudes de los primeros dos eslabones. Mientras que Tras y Rot corresponden a las matrices de transformación homogénea de traslación y rotación con respecto a los ejes indicados.

En la Figura 5 se muestra el robot háptico utilizado (Phantom omni), así como los parámetros y las variables articulares que caracterizan su movimiento y permiten conocer la ubicación de su extremo final (punto de interacción).

Una vez calculada la posición del efector final del robot y conociendo el área de trabajo del mismo, es posible re-escalar esta área de trabajo para que sea del mismo tamaño que el área de trabajo de los objetos virtuales previamente establecida. Asimismo es posible dibujar una representación virtual del efector final, al cual llamaremos puntero, para que el usuario pueda ver la posición en el espacio del robot por medio de los lentes de inmersión. Por lo tanto, el espacio de trabajo virtual tendrá una correspondencia adecuada con el espacio de trabajo háptico definido. Una vez que se tienen superpuestos estos dos espacios de trabajo, se dibujarán objetos hápticos con las mismas características de localización y tamaño que los objetos virtuales, con la diferencia que éstos no estarán diseñados para ser vistos, sino para ser "tocados" por el usuario a través del robot.

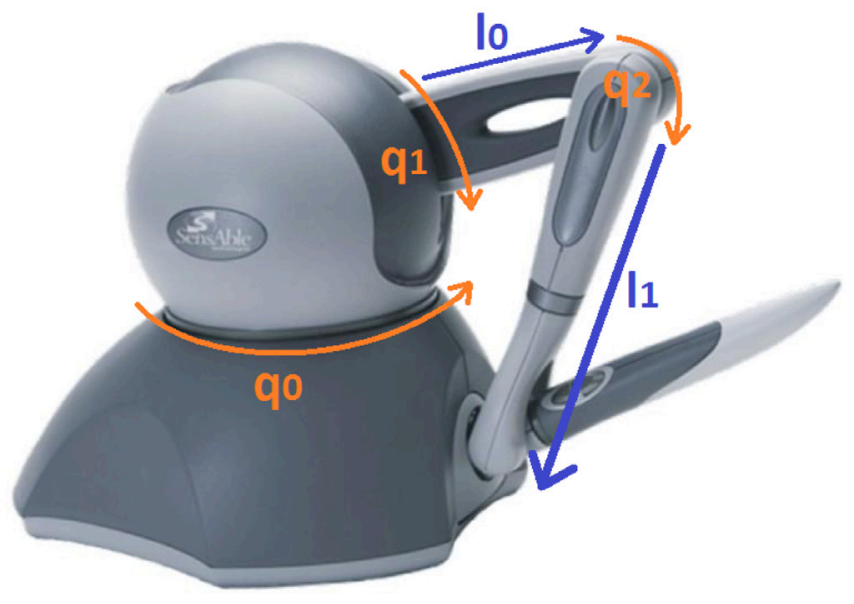

FIGURA 5. Robot háptico.

Otra característica importante del sistema de terapia presentado en este trabajo es la simulación de aspectos físicos como contacto, fricción o peso, de los cuales depende que el usuario tenga una sensación de interacción con objetos virtuales más cercana a la realidad. A continuación se detalla cómo se llevan a cabo dichas simulaciones. 


\section{Contacto}

Es ampliamente conocido que cuando un objeto entra en contacto con una superficie, se genera una fuerza perpendicular a la superficie, dicha fuerza es la encargada de impedir el traslape de los cuerpos ${ }^{[33]}$. Con base en esta definición, es posible entender cómo se lleva a cabo la simulación de contacto. En la Figura 6(a) se observa un cuadro gris que representa el objeto que se desea tocar y un circulo bicolor que representa la posición del efector final del robot háptico, que está compuesto por dos partes: la sección roja es el puntero y representa propiamente la posición del extremo final, mientras que la segunda en color verde, es un componente llamado SCP (Surface Contact Point), el cual se puede describir como un elemento que intenta seguir en todo momento al puntero, pero que a diferencia de éste no puede atravesar la superficie de los objetos con los que se desea interactuar.

a)

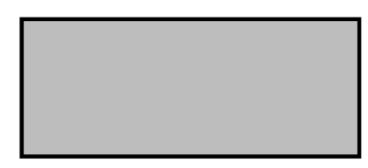

FIGURA 6. Principio de simulación de contacto.

(a) Puntero de la interfaz háptica y

(b) Puntero en contacto con el objeto.

En la Figura 6(b) se observa el comportamiento de los dos elementos que componen la representación del efector final del robot cuando están en contacto con un objeto. A partir de ésto y utilizando la ley de Hook [33], es posible calcular la fuerza de contacto entre el robot y el objeto virtual como se muestra en la Ecuación (13)

$$
F_{c}=-k x_{c}
$$

donde $k$ es el coeficiente de rigidez del objeto que se está tocando y $x_{c}$ es la distancia entre el puntero y el SCP. De esta forma se obtiene la magnitud de la fuerza requerida para simular el contacto entre el extremo

final del robot y el objeto virtual, la cual debe aplicarse en la dirección opuesta al vector formado entre el SCP y el puntero.

\section{Fricción}

Por definición, la fricción es una fuerza que se opone al desplazamiento de dos cuerpos que se encuentran en contacto y es proporcional a la fuerza de contacto presente entre ellos ${ }^{[33]}$.

a)

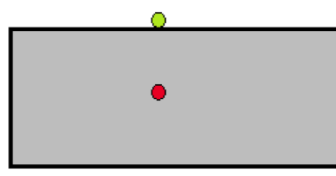

FIGURA 7. Principio de simulación de fricción.

(a) Simulación en el tiempo $t_{0} y$

(b) Simulación en el tiempo t'1

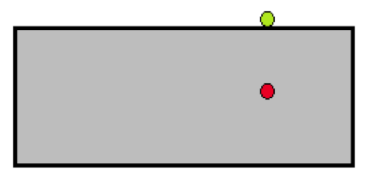

b)

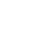


rehabilitación similares y en una serie de pruebas experimentales demuestran la efectividad de los mismos. El escenario virtual diseñado consta de cinco esferas colocadas en diferentes posiciones y a diferentes alturas, que gracias al sistema de realidad aumentada es posible observarlas como si estuvieran presentes en una superficie del escenario real. Así mismo con el uso del robot háptico el usuario del sistema podrá "tocar" las esferas virtuales durante el desarrollo del ejercicio de terapia que se describe a continuación. En un inicio todas las esferas son del mismo color, cuando el ejercicio comienza una de las esferas es elegida de forma aleatoria para cambiar de color, lo que le indica al usuario que debe tocar dicha esfera, al producirse este contacto la esfera regresará a su color original y otra esfera cambiará de color, el ejercicio se repetirá tantas veces como el terapeuta indique.

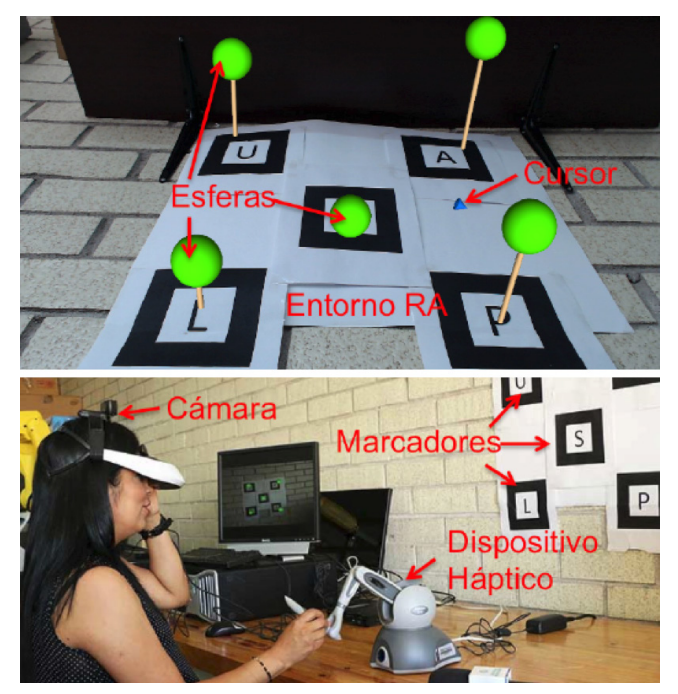

FIGURA 8. Sistema de rehabilitación basado en RA.

Con la finalidad de hacer el sistema más robusto se hace uso de un patrón multimarca (ver Figura 8), esto es, se utilizan cinco marcadores en una distribución específica, logrando que el aumento de realidad sea posible aunque existan oclusiones en todos los marcadores excepto uno. Además, si más de un marcador es visible para la cámara, será tomado como referencia aquel marcador que sea visible de forma más clara, por lo que se logra un aumento de la realidad más fiable.
En los sistemas de realidad aumentada existe un efecto llamado jitter, el cual es generado por señales de ruido interno de las cámaras. Este fenómeno se manifiesta como pequeñas variaciones de la posición en que la cámara detecta el marcador, esto a pesar de que tanto la cámara como el marcador se encuentren fijos. Estas pequeñas variaciones se ven reflejadas en el sistema de aumento de la realidad como vibraciones en los objetos virtuales, situación que puede causar desorientación en los usuarios del sistema.

Con la finalidad de minimizar el efecto de jitter se agregó al sistema un filtro pasa-bajos del tipo promediador con lo que se minimiza la vibración de los objetos virtuales, logrando con esto que dichos elementos sean visualizados como objetos fijos.

Adicionalmente, el sistema fue diseñado para tener la capacidad de exportar información en formato .txt para su análisis posterior. Datos relevantes como la posición del efector final del robot, el número de repeticiones, el tiempo de ejecución del ejercicio, etc. son exportados para realizar un análisis cuantitativo del desempeño de los pacientes durante el ejercicio.

\section{Análisis de datos}

Los datos exportados por el sistema permiten analizar el desempeño de los sujetos de prueba, logrando con esto determinar el grado de mejoría que estos puedan tener al realizar la terapia de rehabilitación.

Como primer método de evaluación es utilizada la norma del error de seguimiento de trayectoria, el cual es un índice de desempeño muy utilizado en el área de la robótica, sirviendo éste como medida comparativa entre la trayectoria ideal que se desea que siga un robot y la trayectoria real seguida por el mismo, la norma del error de seguimiento está dada por

$$
L_{2}=\sqrt{\frac{1}{T} \int_{0}^{T}\|e(t)\|^{2} d t}
$$


donde $T$ es el tiempo total de la prueba y $e(t)$ es el error entre la trayectoria seguida y la trayectoria ideal que, para el caso del ejercicio de terapia ocupacional propuesto, es una línea recta.

Otra forma evaluar el desempeño de los usuarios del sistema propuesto, al realizar ejercicios de rehabilitación, es evaluando su condición muscular; esto puede llevarse acabo analizando señales de EMG de los músculos ejercitados durante la terapia. La selección de parámetros o índices para analizar las señales de EMG se realizó de tal forma que éstos brindaran información suficiente para determinar un posible fortalecimiento de los músculos ejercitados, o en su defecto verificar que los pacientes no sufrieran fatiga muscular a consecuencia del uso del sistema.

Se seleccionaron dos índices en el domino del tiempo, como son el valor RMS y la longitud de onda, definidos como

$$
\begin{aligned}
& R M S=\sqrt{\frac{1}{M} \sum_{m=1}^{M} x_{m}^{2}} \\
& w L=\sum_{m=1}^{M-1}\left|x_{m+1}-x_{m}\right|
\end{aligned}
$$

donde $x_{m}$ representa la muestra $m$ de la señal de EMG, $M$ el número total de muestras de la señal. El valor RMS brinda información de la potencia promedio de la señal EMG y el parámetro $w L$ aporta información referente a la energía y la complejidad de la señal muscular ${ }^{[21]}$.

Es importante señalar que estos parámetros fueron seleccionados debido a su baja complejidad de cómputo, pudiendo calcularse incluso en tiempo real, y además aportan información similar a la de parámetros como Zero Crossing Rate (ZCR) o Slope Sign Change (SSC), siendo uno de los más robustos ante ruido en la señal EMG ${ }^{[22]}[23]$.
Asimismo, buscando tener un panorama más amplio de las características que una señal EMG puede aportar, se consideraron dos índices en el dominio de la frecuencia: la potencia total del espectro (PT) y la frecuencia mediana (MDF) dadas por

$$
\begin{gathered}
P T=\sum_{n=1}^{N} P_{n} \\
M D F=\frac{1}{2} \sum_{n=1}^{N} P_{n}
\end{gathered}
$$

donde $P_{n}$ es la muestra n del espectro de la señal y $N$ es la longitud total del espectro. La PT brinda información acerca de la fuerza muscular, mientras que la $M D F$ aporta información acerca de la fatiga muscular de los pacientes ${ }^{[35]}$; siendo estos índices algunos de los más comunes ${ }^{[22]}$, lo que facilita comparar los resultados obtenidos con los de trabajos previos, además de que han presentado un alto desempeño en estudios previos ${ }^{[23]}$.

El protocolo utilizado para la obtención de las señales de EMG fue el siguiente: se le pidió al paciente relajar el músculo del que se está registrando la señal para establecer un nivel de referencia, posteriormente se deben realizar cinco series compuestas de una contracción máxima del músculo durante cinco segundos y posteriormente un periodo de relajación de diez segundos, lo anterior se realiza antes y después de realizar la terapia de rehabilitación. Cabe señalar que cada una de las sesiones de rehabilitación tuvo una duración aproximada de 30 minutos, llevándose a cabo un número de repeticiones determinado por el terapeuta.

\section{Selección de sujetos de prueba}

El sistema de terapia fue probado con 10 pacientes, de entre 4 y 14 años de edad, con una edad promedio de 7.83 años y una desviación estándar de 3.97. El proceso de selección fue realizado por un terapeuta ocupacional que trabajaba regularmente con los pacientes, considerando a quienes requerían mejorar su capacidad de 
manipulación fina en extremidades superiores. Los tipos de discapacidad de los pacientes son: artrogriposis (2 sujetos), síndrome de Guillain Barré (2 sujetos), amputación de muñeca (2 sujetos), amputación de codo (2 sujetos) y lesión del plexo braquial (2 sujetos). Cabe señalar que todos los pacientes participaron en las terapias de forma voluntaria sin remuneración económica, habiendo firmado previamente un consentimiento informado por parte de los tutores de los mismos y siempre bajo la supervisión de éstos.

\section{RESULTADOS Y DISCUSIÓN}

En primer lugar se llevó a cabo una sesión de entrenamiento, en la cual se les explicó a los sujetos de prueba el objetivo de la terapia y que además les permitió familiarizarse con el sistema, lo anterior siempre bajo la supervisión de sus tutores y su terapeuta. A continuación se describen los resultados obtenidos en las pruebas experimentales realizadas una vez concluida la sesión de familiarización.

\section{Análisis y evaluación de seguimiento de trayectoria durante la terapia}

Inicialmente se evaluó la funcionalidad del sistema con base en la trayectoria seguida por los pacientes al realizar los ejercicios de rehabilitación, comparando los resultados obtenidos en la primera sesión de rehabilitación y después de un cierto número de sesiones. Es decir, se grafican las trayectorias realizadas y se hace una comparación de los comportamientos obtenidos, de esta forma es posible determinar si existió o no mejoría en el desempeño del paciente, a partir de la precisión y suavidad de los movimientos realizados. Debido a que la comparación visual de las trayectorias seguidas por los pacientes al realizar el ejercicio puede ser un criterio subjetivo, se utilizan datos cuantitativos para validar si existió una mejoría en los pacientes y cuantificar el grado de dicha mejoría. Como parámetros de cuantificación fueron utilizados el tiempo de ejecución del ejercicio y la norma del error de seguimiento de trayectoria, calculada por medio de la Ecuación (15).
En la Figura 9 se grafican las trayectorias seguidas por tres de los sujetos de prueba al llevar a cabo el ejercicio de rehabilitación, en amarillo se representa la superficie sobre la cual son proyectadas las cinco esferas virtuales. En las Figuras 9(a), 9(c) y 9(e) se observa el desempeño que tuvieron los tres sujetos de prueba al realizar la primera sesión del ejercicio, en las Figuras 9(b), 9(d) y 9(f) se observan las trayectorias seguidas por los mismos sujetos en la sesión número 20. Es claro que para todos los casos en la primera sesión la trayectoria seguida tiene una conducta más errática, siendo notoria la mejoría de cada uno de ellos después de culminar todas las sesiones de rehabilitación.

En la Tabla 1 se presentan los resultados cuantitativos de cada uno de los sujetos de prueba, en todos los casos se puede observar una mejora en el tiempo de ejecución del ejercicio, reduciendo este tiempo en aproximadamente $60 \%$ para el primer paciente y del $38 \%$ para los otros dos. Asimismo, el promedio del error se redujo considerablemente, siendo esa mejora entre el 50\% y el 60\% aproximadamente.

TABLA 1. Tiempo de ejecución y norma del error.

\begin{tabular}{|l|c|c|c|c|}
\hline & \multicolumn{2}{|c|}{$\begin{array}{c}\text { Tiempo de } \\
\text { ejecución (s) }\end{array}$} & \multicolumn{2}{c|}{$\begin{array}{c}\text { Error } \\
(\mathrm{cm})\end{array}$} \\
\hline & \multicolumn{2}{|c|}{ Número de sesión } & \multicolumn{2}{c|}{ Número de sesión } \\
\hline & 1 & 20 & 1 & 20 \\
\hline Sujeto 1 & 114.001 & 46.053 & 4.2018 & 1.8902 \\
\hline Sujeto 2 & 108.214 & 67.487 & 5.4969 & 2.1851 \\
\hline Sujeto 3 & 82.0970 & 51.205 & 11.3883 & 5.5640 \\
\hline
\end{tabular}

En la Figura 10 se presentan las gráficas de la norma del error contra el tiempo de ejecución del ejercicio, donde cada uno de los círculos en la gráfica representa el instante donde se produce el contacto con la esfera virtual que se debe tocar. 
a)

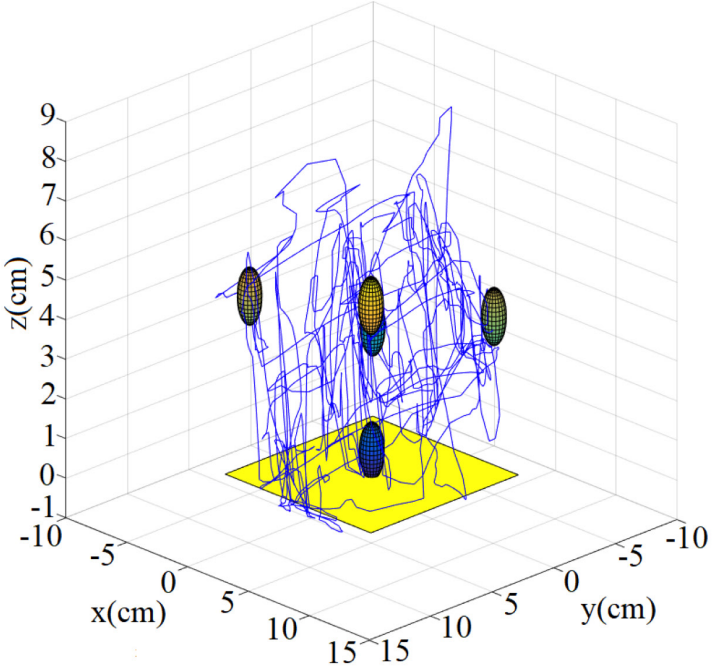

C)

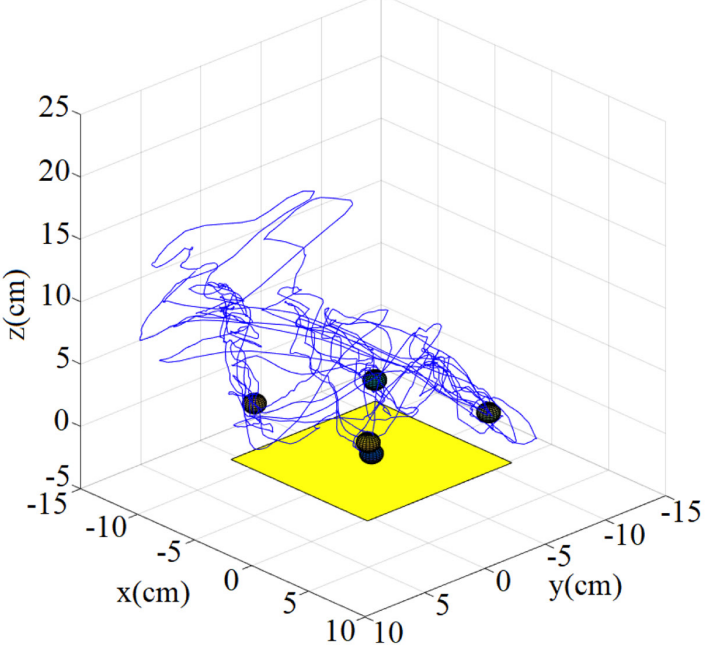

e)

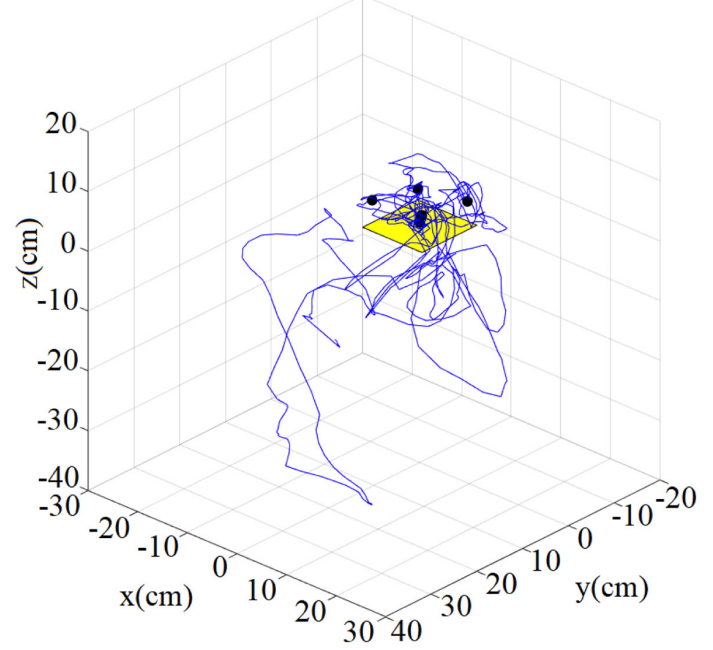

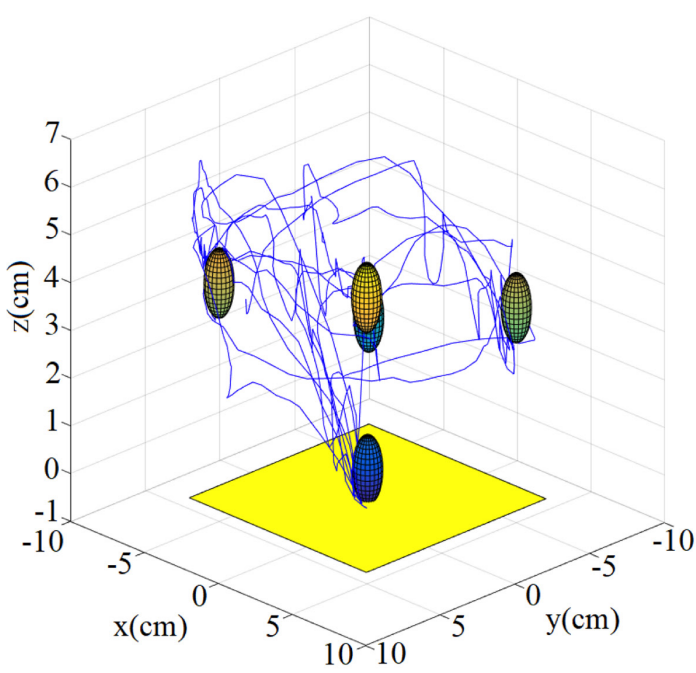

b)

d)

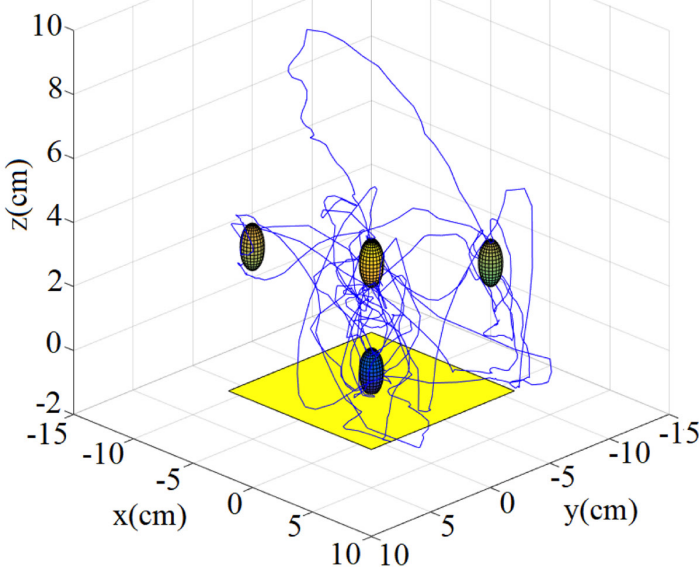

f)

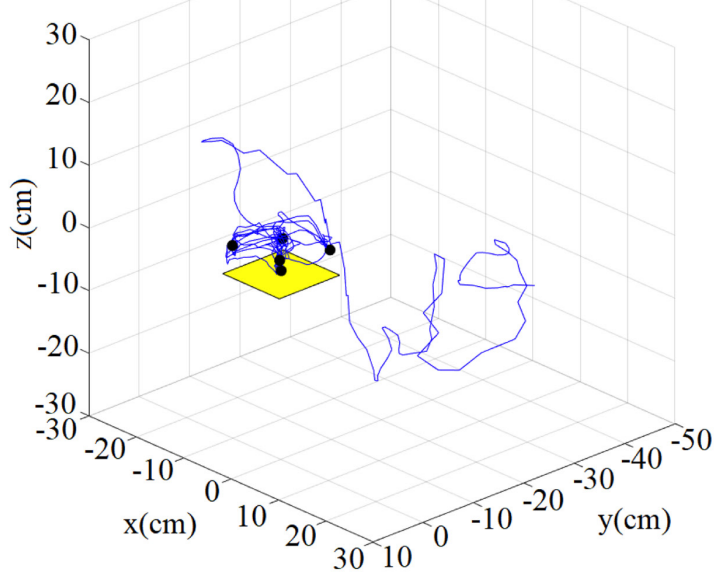

FIGURA 9. Trayectorias seguidas por los pacientes. (a) Sujeto 1- sesión inicial, (b) Sujeto 1- sesión final, (c) Sujeto 2- sesión inicial, (d) Sujeto 2- sesión final, (e) Sujeto 3- sesión inicial y (f) Sujeto 3- sesión final. 

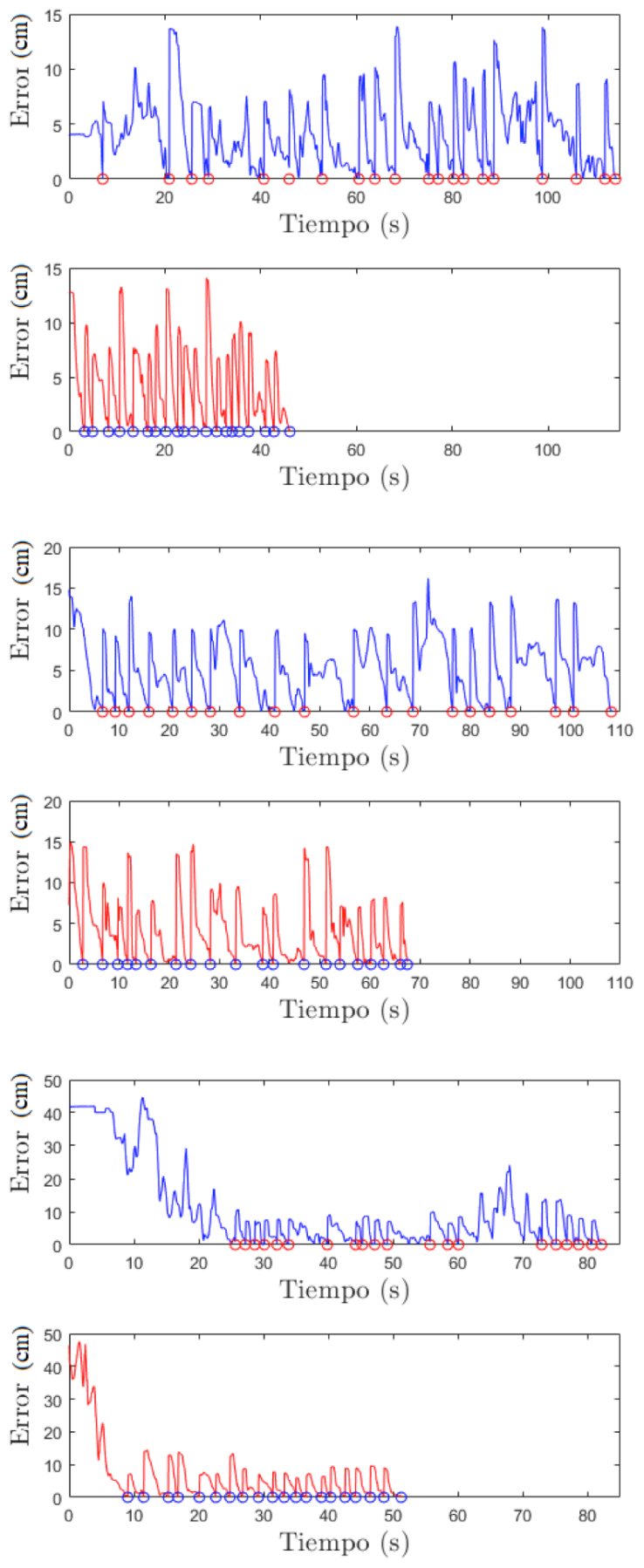

FIGURA 10. Norma del error de trayectoria.

(a) Sujeto 1, (b) Sujeto 2 y (c) Sujeto 3.

Se observa que una vez producido el contacto el error crece súbitamente, debido a que en ese instante cambia la esfera que se debe tocar, existiendo entonces una distancia entre la posición del cursor y la nueva esfera que se debe tocar, lo cual incrementa el valor del error.
En color azul se presentan las gráficas de los tres sujetos de prueba en la primera sesión de terapia y en color rojo las gráficas de la sesión número 20; en todos los casos es posible notar cómo el error decrece de una forma más suave y con un tiempo de ejecución mucho menor en la última sesión de rehabilitación.

\section{Evaluación de la condición muscular}

Para esta segunda etapa de evaluación del sistema de terapia, además de los criterios de trayectoria seguida por el paciente y la norma del error de seguimiento de trayectoria utilizados en la etapa anterior, se evalúa

b) la condición muscular de los pacientes. Para este fin se registran señales EMG con el protocolo descrito previamente y se hace una evaluación de las señales obtenidas utilizando las Ecuaciones (16), (17), (18) y (19).

La duración de esta segunda etapa de pruebas fue de tres meses, donde cada uno de los pacientes asistió a dos sesiones de terapia semanales. Los resultados aquí presentados corresponden a muestras tomadas en cada uno de los pacientes en la primera y en la última sesión de rehabilitación. De manera ilustrativa se presentan los resultados de los dos sujetos de prueba que fueron más constantes durante el periodo de evaluación.

El primer sujeto de prueba fue diagnosticado con artrogriposis, por lo que el músculo a evaluar y sobre el que se tomaron las señales de EMG fue el músculo flexor, ya que éste es el responsable del agarre de la mano con la que el paciente sujeta el sistema háptico. Por otra parte, el diagnóstico del sujeto dos es amputación transcarpiana (desarticulado de muñeca), por lo que el músculo a evaluar y donde se realizan las mediciones de EMG es el deltoides, ya que éste es el encargado del movimiento de su prótesis.

En la Figura 11 se observan las gráficas de trayectorias seguidas por ambos sujetos de prueba en la primera (Figuras 11(a) y 11(c)) y en la última sesión de terapia (Figuras 11(b) y 11(d)), de igual forma que con los resul- 
a)

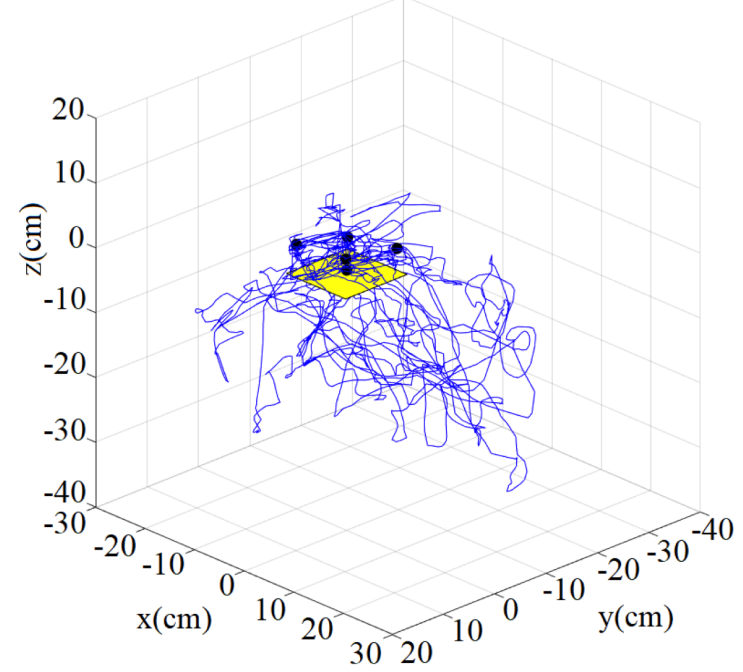

C)

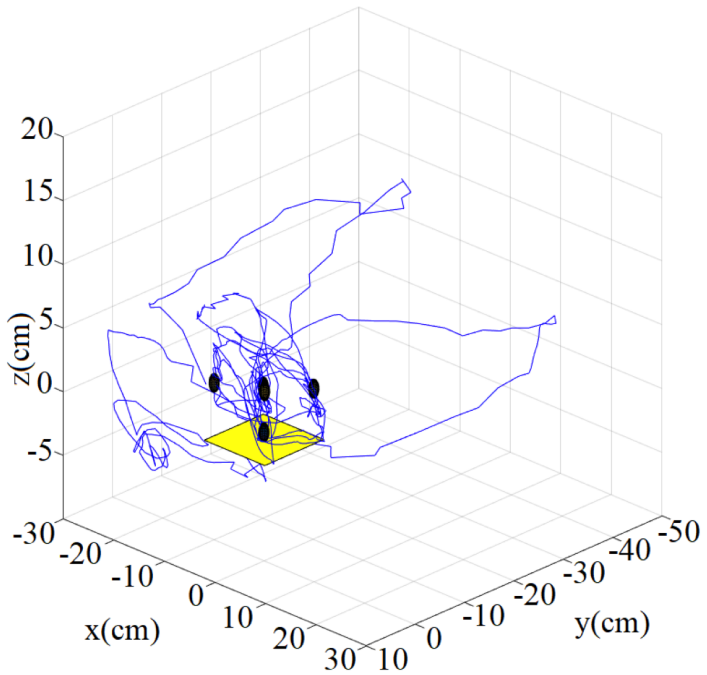

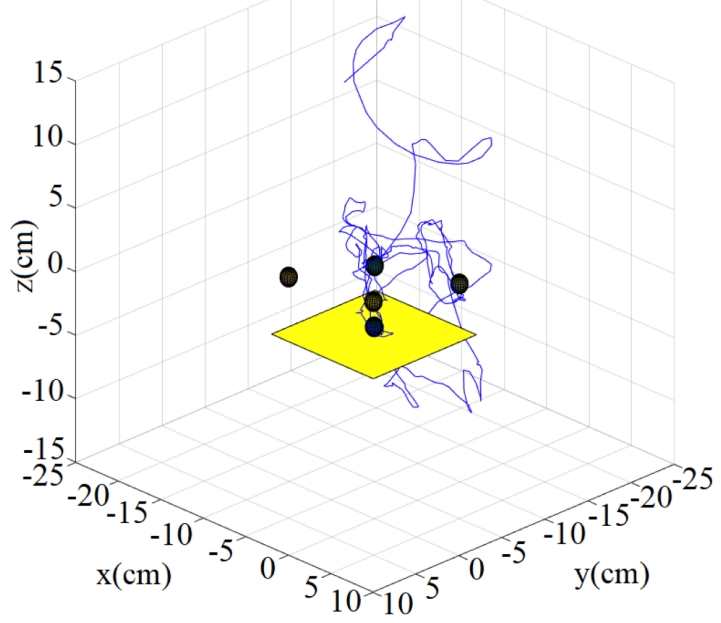

b)

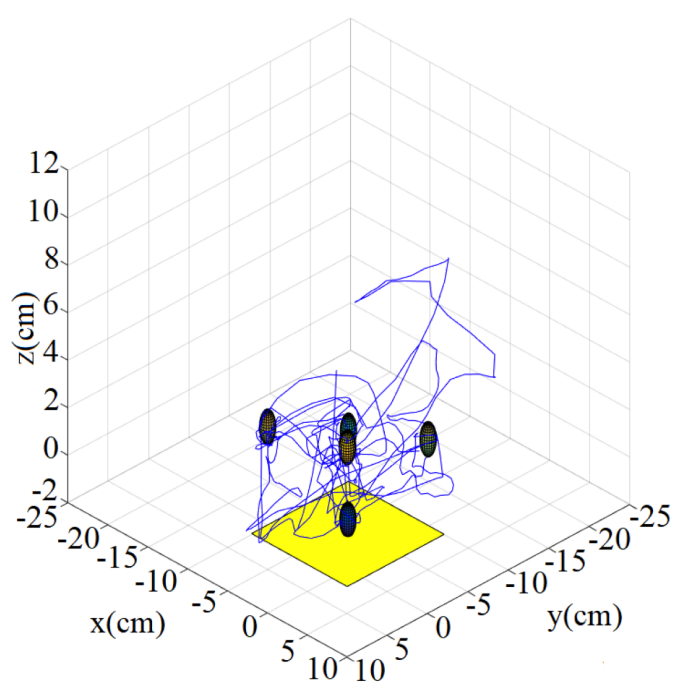

d)

FIGURA 11. Trayectorias seguidas por los pacientes. (a) Sujeto 1- sesión inicial, (b) Sujeto 1- sesión final,

(c) Sujeto 2- sesión inicial, (d) Sujeto 2- sesión final.

tados de los pacientes mostrados en la sección anterior, existe una mejoría notable después de las sesiones de rehabilitación, lo cual indica un mayor control en los movimientos finos de todos los pacientes. De igual forma se muestran las gráficas de error de ambos sujetos de prueba (ver Figura 12), donde también se puede observar un tiempo mucho menor al realizar el ejercicio, amplitudes de error menores y una disminución del error con respecto al tiempo mucho más suave si se comparan la primera y la última sesión de terapia.

Por último, se presentan los resultados del análisis de la señal de EMG para el sujeto uno (ver tabla 2), éstos son congruentes con lo que se podría esperar, es decir, tanto en la primera como en la última sesión de terapia se puede observar una disminución en los índices calculados después de la terapia de rehabilitación, lo cual indica cierto grado de fatiga muscular. Haciendo una comparativa de los índices calculados entre la primera y la última sesión, se observa un aumento en todos los parámetros, lo cual indica un fortalecimiento de los músculos que se ejercitaron con la terapia.

En la tabla 3 se observan los datos obtenidos de la señal de EMG del segundo sujeto de prueba. En este caso los resultados no concuerdan con lo esperado, 


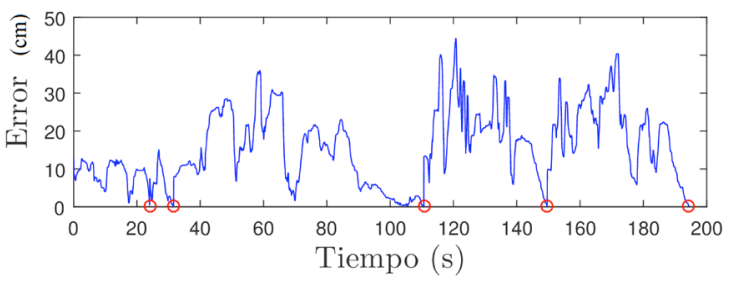

a)
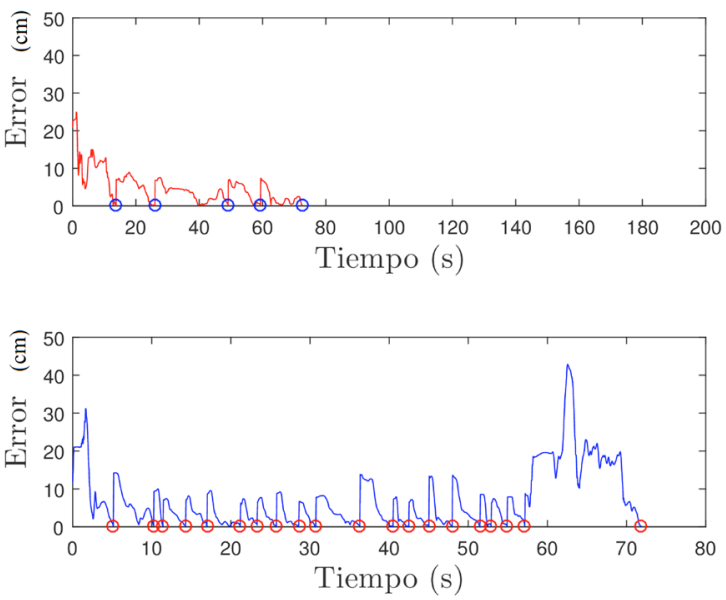

b)

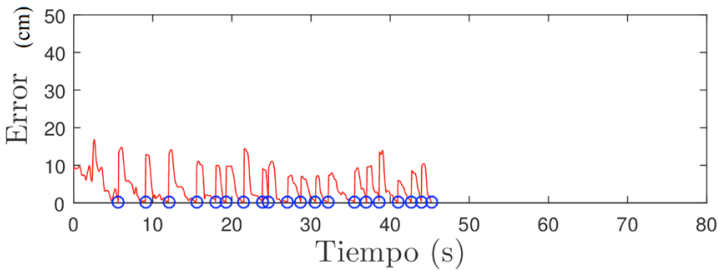

FIGURA 12. Norma del error de trayectoria.

(a) Sujeto 1, (b) Sujeto 2.

una posible explicación a los resultados obtenidos es que debido a la dificultad propia de la condición del paciente, este no podía realizar las contracciones mus-

TABLA 2. Índices calculados para el sujeto de prueba 1 (AT: antes de la terapia, DT: después de la terapia).

\begin{tabular}{|l|c|c|}
\hline \multirow{2}{*}{\multicolumn{1}{|c|}{ Índice }} & \multicolumn{2}{|c|}{ Primera sesión } \\
\cline { 2 - 3 } & AT & DT \\
\hline PT (HZ) & $30378 \pm 1348$ & $26982 \pm 270$ \\
\hline MDF (Hz) & $256.49 \pm 1.27$ & $249.39 \pm 1.6$ \\
\hline RMS (V) & 0.1957 & 0.1800 \\
\hline \multirow{2}{*}{ wL (V) } & 550.3690 & 500.1089 \\
\hline \multirow{2}{*}{ Índice } & \multicolumn{2}{|c|}{ Última sesión } \\
\cline { 2 - 3 } & AT & DT \\
\hline PT (HZ) & $38892 \pm 58.9$ & $432230 \pm 976.8$ \\
\hline MDF (Hz) & $228.29 \pm 0.98$ & $222.59 \pm 6.5$ \\
\hline RMS (V) & 0.2579 & 0.2893 \\
\hline wL (V) & 744.3286 & 859.7678 \\
\hline
\end{tabular}

culares de la forma que se le solicitó, por lo que se tuvo que reducir el periodo de análisis de la señal, pudiendo con esto afectar los resultados de la prueba.

TABLA 3. Índices calculados para el sujeto de prueba 2 (AT: antes de la terapia, DT: después de la terapia).

\begin{tabular}{|l|c|c|}
\hline \multirow{2}{*}{\multicolumn{1}{|c|}{ Índice }} & \multicolumn{2}{|c|}{ Primera sesión } \\
\cline { 2 - 3 } & AT & DT \\
\hline PT (HZ) & $7366 \pm 1308$ & $5939.7 \pm 712$ \\
\hline MDF (Hz) & $73.24 \pm 15.9$ & $81.49 \pm 3.53$ \\
\hline RMS (V) & 0.2614 & 0.2614 \\
\hline \multirow{2}{*}{ wL (V) } & 140.2341 & 155.54 \\
\hline \multirow{2}{*}{ Índice } & \multicolumn{2}{|c|}{ Última sesión } \\
\cline { 2 - 3 } & AT & DT \\
\hline PT (HZ) & $2488.1 \pm 14.6$ & $3265 \pm 487.45$ \\
\hline MDF (Hz) & $78.74 \pm 0.35$ & $85.99 \pm 4.24$ \\
\hline RMS (V) & 0.0957 & 0.1375 \\
\hline wL (V) & 61.1777 & 79.5895 \\
\hline
\end{tabular}




\section{Valoración de los pacientes por parte de un terapeuta ocupacional}

Con la finalidad de dar mayor validez a los resultados cuantitativos aquí presentados, se le pidió a un terapeuta valorar el estado general de los pacientes que utilizaron el sistema; la valoración se realizó antes y después de las sesiones de terapia, las conclusiones de este análisis se presentan a continuación.

De manera general, el terapeuta coincide que después de utilizar el sistema todos los pacientes tuvieron mejorías en aspectos físicos de las extremidades superiores como aumento del tono muscular y del rango de movimiento, mejoras notables en la coordinación gruesa y fina, así como mejora en la fuerza, destreza, resistencia motriz y habilidades visomotoras.

En opinión del terapeuta los cambios logrados en los sujetos de prueba no solamente son perceptibles en el aspecto físico, si no que también se lograron mejoras en aspectos cognitivos y psicológicos, tales como mejoras en el procesamiento cognitivo, logrando respuestas adecuadas en resolución de problemas de mayor complejidad de acuerdo a la edad de los pacientes, aumento en la capacidad de aprendizaje, buena disposición para participar de las terapias, aumento en el autoreconocimiento de los avances logrados, así como mayor autoaceptación de su condición física.

Por otra parte, se observó el comportamiento de los participantes para determinar su nivel de satisfacción, ya que por su corta edad se consideró poco confiable realizarles una encuesta. En el análisis, se pudo observar una gran disposición de participar en las sesiones, aunque, por la naturaleza repetitiva de los ejercicios y la corta edad de los pacientes, estos mostraban aburrimiento entre los 10 y 15 minutos de utilizar el sistema.

Por último, es importante mencionar que los sujetos de prueba mostraron cierta incomodidad e incluso mareos al usar los lentes de inmersión, por lo que todos prefirieron realizar las sesiones de terapia utilizando como medio de visualización un monitor. Cabe señalar que durante el periodo de prueba del sistema, éste fue utilizado por otro grupo de personas (mayores de edad), coincidiendo todos que una de las características más atractivas del sistema era la posibilidad de ver los objetos reales mezclados con virtuales a través de los lentes de inmersión, por lo que se puede pensar que la negativa de utilizar los lentes por parte de los sujetos de prueba fue debido al tamaño de los mismos y no a su funcionamiento.

\section{CONCLUSIONES}

Se desarrolló un sistema novedoso en el área de rehabilitación robótica, principalmente enfocado a la rehabilitación de movimientos finos de extremidades superiores, cuya principal característica es la posibilidad de interactuar de forma visual y táctil con objetos virtuales mezclados con escenarios del mundo real. La característica mencionada previamente hace de éste un sistema muy versátil, ya que permite realizar modificaciones a la terapia de rehabilitación, generando con esto distintas rutinas de rehabilitación con diferentes grados de dificultad sin la necesidad de equipo adicional al mencionado previamente.

En pruebas realizadas con pacientes con distintos tipos de condiciones que dificultan los movimientos de sus extremidades superiores, fue posible observar el potencial que tiene el sistema como herramienta auxiliar a las terapias de rehabilitación tradicional al permitir guardar los datos generados por los usuarios en cada una de sus terapias, destacando que en la mayoría de los casos, el análisis cuantitativo de dichos datos muestran mejoras significativas en el control de los movimientos del paciente, disminución del tiempo de ejecución en los ejercicios y fortalecimiento muscular.

Un punto importante a resaltar, es que las sesiones de rehabilitación robótica fueron supervisadas por el terapeuta de los sujetos de prueba, realizando evalua- 
ciones de los mismos antes y después de las sesiones de terapia, concluyendo que el uso del sistema ayudó a los pacientes al mejorar características como fuerza, destreza, resistencia, coordinación, rango de movimiento, etc., lo cual muestra el potencial del sistema como herramienta de rehabilitación.

Según los terapeutas, una de las situaciones que propician que un paciente no continúe con un programa de rehabilitación es el tedio producido por la constante repetición de los ejercicios. El sistema presentado tiene la característica de incentivar a los pacientes a continuar con sus terapias por medio de un sistema de rehabilitación novedoso, lo cual quedó demostrado, ya que los pacientes mostraron entusiasmo al utilizar el sistema y estar presentes en todas las sesiones de terapia.

Es importante mencionar que una de las principales limitantes con las que cuenta actualmente el sistema propuesto es que sólo procesa imágenes de forma bidimensional, de manera que tanto las imágenes virtua- les como las del mundo real son proyectadas al usuario sin que éste pueda percibir de forma adecuada la profundidad de la escena, lo que dificulta el proceso de familiarización por parte de los usuarios. Así mismo, los resultados obtenidos en esta primera fase experimental han sido limitados por la disponibilidad, la cantidad y la variedad de padecimientos de los sujetos de prueba evaluados; esperando a futuro contar con una muestra más grande y homogénea.

Por último es conveniente mencionar que el sistema presentado demuestra potencial para ser una herramienta útil como complemento a las terapias de rehabilitación convencionales, debiéndose realizar pruebas experimentales con un grupo mayor de sujetos de prueba para corroborar los datos obtenidos hasta el momento, así como desarrollar un número mayor de terapias de rehabilitación virtuales con ayuda de terapeutas para aumentar el interés por parte de los pacientes en el sistema y propiciar el no abandono de sus terapias. 


\section{REFERENCIAS}

[1] Banco Mundial, "Informe mundial sobre la discapacidad," Organización Mundial de la Salud (2011), 2011.

[2] Instituto Nacional de Estadística y Geografía, "La discapacidad en México. Datos al 2014.,” Publicaciones INEGI, 2016.

[3] World Health Organization, "The world health report 2006: working together for health,” 2006.

[4] K. B. Valles, S. Montes, M. J. Madrigal, A. Burciaga, M. E. Martínez, and M. J. Johnson, "Technology-assisted stroke rehabilitation in México: a pilot randomized trial comparing traditional therapy to circuit training in a robot/technology-assisted therapy gym," Journal of NeuroEngineering and Rehabilitation, vol. 13, no. 1, p. 83, 2016. DOI: 10.1186/s12984-016-0190-1

[5] I. Díaz, J. J. Gil, and E. Sánchez, "Lower-limb robotic rehabilitation: literature review and challenges,” Journal of Robotics, vol. 2011, 2011.

[6] N. Yozbatiran, J. Berliner, M. K. O’Malley, A. U. Pehlivan, Z. Kadivar, C. Boake, and G. E. Francisco, "Robotic training and clinical assessment of upper extremity movements after spinal cord injury: a single case report," Journal of rehabilitation medicine, vol. 44, no. 2, pp. 186-188, 2012. DOI: 10.2340/16501977-0924

[7] A. Borboni, M. Mor, and R. Faglia, "Gloreha - hand robotic rehabilitation: design, mechanical model, and experiments," Journal of Dynamic Systems, Measurement, and Control, vol. 138, no. 11, p. 111003, 2016. DOI: 10.1115/1.4033831

[8] J. Sivenius, K. Pyörälä, O. Heinonen, J. Salonen, and P. Riekkinen, "The significance of intensity of rehabilitation of stroke-a controlled trial.," Stroke, vol. 16, no. 6, pp. 928-931, 1985. DOI: 10.1161/01.STR.16.6.928

[9] G. Kwakkel, R. C. Wagenaar, T. W. Koelman, G. J. Lankhorst, and J. C. Koetsier, "Effects of intensity of rehabilitation after stroke," Stroke, vol. 28, no. 8, pp. 1550-1556, 1997. DOI: 10.1161/01. STR.28.8.1550

[10] N. Barrett, I. Swain, C. Gatzidis, and C. Mecheraoui, "The use and effect of video game design theory in the creation of game-based systems for upper limb stroke rehabilitation," Journal of Rehabilitation and Assistive Technologies Engineering, vol. 3, pp. 1-16, 2016. DOI: 10.1177/2055668316643644

[11] F. D. Rose, B. M. Brooks, and A. A. Rizzo, "Virtual reality in brain damage rehabilitation: review," Cyberpsychology \& behavior, vol. 8, no. 3, pp. 241-262, 2005. DOI: 10.1089/cpb.2005.8.241

[12] D. Jack, R. Boian, A. S. Merians, M. Tremaine, G. C. Burdea, S. V. Adamovich, M. Recce, and H. Poizner, "Virtual reality-enhanced stroke rehabilitation," IEEE transactions on neural systems and rehabilitation engineering, vol. 9, no. 3, pp. 308-318, 2001. DOI: $10.1109 / 7333.948460$

[13] J. H. Shin, H. Ryu, and S. H. Jang, "A task-specific interactive gamebased virtual reality rehabilitation system for patients with stroke: a usability test and two clinical experiments," Journal of neuroengineering and rehabilitation, vol. 11, no. 1, p. 32, 2014. DOI: 10.1186/1743-0003-11-32

[14] G. Saposnik, M. Mamdani, M. Bayley, K. Thorpe, J. Hall, L. Cohen, and R. Teasell, "Effectiveness of virtual reality exercises in stroke rehabilitation (evrest): rationale, design, and protocol of a pilot randomized clinical trial assessing the wii gaming system," International Journal of Stroke, vol. 5, no. 1, pp. 47-51, 2010. DOI: 10.1111/j.1747-4949.2009.00404.x
[15] G. Saposnik, M. Levin, S. O. R. C. S. W. Group, et al., "Virtual reality in stroke rehabilitation,” Stroke, vol. 42, no. 5, pp. 1380-1386, 2011. DOI: 10.1161/STROKEAHA.110.605451

[16] V. C. L. Chiang, K.-H. Lo, and K. S. Choi, "Rehabilitation of activities of daily living in virtual environments with intuitive user interface and force feedback," Disability and Rehabilitation: Assistive Technology, pp. 1-9, 2016. DOI: $10.1080 / 17483107.2016 .1218554$

[17] A. Turolla, O. A. Daud Albasini, R. Oboe, M. Agostini, P. Tonin, S. Paolucci, G. Sandrini, A. Venneri, and L. Piron, "Haptic-based neurorehabilitation in poststroke patients: a feasibility prospective multicentre trial for robotics hand rehabilitation," Computational and mathematical methods in medicine, vol. 2013, 2013. DOI: $10.1155 / 2013 / 895492$

[18] C. González, D. Vallejo, J. Albusac, and J. Castro, "Realidad aumentada. Un enfoque práctico con artoolkit y blender,” 2013.

[19] M. S. Hossain, S. Hardy, A. Alamri, A. Alelaiwi, V. Hardy, and C. Wilhelm, "Ar-based serious game framework for post-stroke rehabilitation,” Multimedia Systems, vol. 22, no. 6, pp. 659-674, 2016. DOI: 10.1007/s00530-015-0481-6

[20] A. Y. Dvorkin, F. S. Zollman, K. Beck, E. Larson, and J. L. Patton, “A virtual environment-based paradigm for improving attention in tbi," in 2009 IEEE International Conference on Rehabilitation Robotics, pp. 962-965, IEEE, 2009. DOl: 10.1109/ ICORR.2009.5209629

[21] A. Phinyomark, G. Chujit, P. Phukpattaranont, C. Limsakul, and H. $\mathrm{Hu}$, "A preliminary study assessing time-domain emg features of classifying exercises in preventing falls in the elderly," in Electrical Engineering/Electronics, Computer, Telecommunications and Information Technology (ECTI-CON), 2012 9th International Conference on, pp. 1-4, IEEE, 2012. DOI: 10.1109/ ECTICon.2012.6254117

[22] Mario Cifrek, Vladimir Medved, and Stanko Tonkovic", "Surface EMG based muscle fatigue evaluation in biomechanics," in Clinical Biomechanics, vol. 24, no. 4, pp. 327-340, 2009. DOI: 10.1016/j. clinbiomech.2009.01.010

[23] Angkoon Phinyomark, S. Hirunviriya, C. Limsakul, and P. Phukpattaranont, "Evaluation of EMG feature extraction for hand movement recognition base on Euclidean distance and standard deviation," in Electrical Engineering/Electronics Computer Telecommunications and Information Technology (ECTI-CON), pp. 856-860, IEEE, 2010.

[24] Reza Boostani, and Mohammad Hassan Moradi, "Evaluation of the forearm EMG signal features for the control of a prosthetic hand," in Physiological measurement, vol. 24, no. 2, pp. 309-321, 2003.

[25] C. Vidrios-Serrano, I. Bonilla, F. Vigueras-Gómez, and M. Mendoza, "Development of a haptic interface for motor rehabilitation therapy using augmented reality," in Engineering in Medicine and Biology Society (EMBC), 2015 37th Annual International Conference of the IEEE, pp. 1156-1159, IEEE, 2015. DOI: 10.1109/ EMBC.2015.7318571

[26] C. Vidrios-Serrano, I. Bonilla, and F. Vigueras-Gómez, "Interfaz háptica para terapias de rehabilitación utilizando realidad aumentada," AMRob Journal, Robotics: Theory and Applications, vol. 3, no. 3, pp. 74-80, 2015. 
[27] A. M. Torres Hernández, B. E. Amaro Amaro, V. I. Ramírez Vera, M. O. Mendoza Gutiérrez, and I. Bonilla Gutiérrez, “Cuantificación del avance en terapia de rehabilitación de miembros superiores mediante el uso de una interfaz háptica y realidad aumentada," in Memorias XXXVIII Congreso Nacional de Ingeniería Biomédica (CNIB), pp. 297-300, Sociedad Mexicana de Ingeniería Biomédica, 2015. DOI: dx.doi.org/10.24254/CNIB.15.48

[28] R. Hartley and A. Zisserman, "Multiple view geometry in computer vision,” Cambridge university press, 2003.

[29] P. Hough, "Method and means for recognizing complex patterns," Dec. 18 1962. US Patent 3,069,654.

[30] G. Simon, A. W. Fitzgibbon, and A. Zisserman, "Markerless tracking using planar structures in the scene,” Augmented Reality, 2000. (ISAR 2000). Proceedings. IEEE and ACM International Symposium on, pp. 120-128, 2000. DOI: 10.1109/ISAR.2000.880935

[31] G. H. Golub and C. F. Van Loan, "Matrix computations," JHU Press, vol. 3, 2012.
[32] F. Reyes Cortes, "Robótica: control de robots manipuladores," 2011.

[33] P. A. Tipler and G. Mosca, "Física para la ciencia y la tecnología," Reverté, vol. 2, 2005.

[34] H. I. Krebs, J. J. Palazzolo, L. Dipietro, M. Ferraro, J. Krol, K. Rannekleiv, B. T. Volpe, and N. Hogan, "Rehabilitation robotics: Performance-based progressive robot-assisted therapy," Autonomous robots, vol. 15, no. 1, pp. 7-20, 2003. DOI: 10.1023/A:1024494031121

[35] S. Thongpanja, A. Phinyomark, P. Phukpattaranont, and C. Limsakul, "Mean and median frequency of emg signal to determine muscle force based on time-dependent power spectrum," Elektronika ir Elektrotechnika, vol. 19, no. 3, pp. 51-56, 2013. DOI: $\underline{10.5755 / j 01 . e e e .19 .3 .3697}$ 